\title{
Clinical Study on Treatment of Patients with Acute Cerebral Infarction by Huayu Tongluo Decoction Combined with Edaravone Injection
}

\author{
Wentao Zhang, Ping Zuo, Feng Wang, Zhihua Liu, Yinglin Cui \\ Henan Province Hospital of Traditional Chinese Medicine, Henan, Zhengzhou, 450002, China
}

Keywords: Cerebral Infarction; Acute Phase; Huatan Tongluo Decoction; Edaravone Injection; Neurological Function; Quality Of Life

\begin{abstract}
Objective: To study the clinical effect of Huayu Tongluo Decoction combined with edaravone injection in the treatment of patients with acute cerebral infarction. Methods: 86 patients with acute cerebral infarction received from our hospital from October 2016 to May 2018 were randomly divided into study group $(n=43)$ and control group $(n=43)$. Routine treatment was performed in both groups. On the basis of this, the control group was treated with edaravone injection, and the research group was treated with Huayu Tongluo Decoction combined with edaravone injection. The effects of treatment, the degree of neurological deficit (NIHSS score), TCM syndrome scores and quality of life (QLI score) before and after treatment were compared. Results: The total effective rate of the study group [95.35\% (41/43)] was higher than that of the control group [79.07\% (34/43)] $(\mathrm{P}<0.05)$. After treatment, the main symptoms, secondary diseases and tongue and pulse of the two groups were treated. The scores were lower than before treatment, and the study group was lower than the control group $(\mathrm{P}<0.05)$. After treatment, the NIHSS and QLI scores of the two groups were better than before treatment, and the NIHSS score of the study group was lower than that of the control group, and the QLI score was higher than the control. Group $(\mathrm{P}<0.05)$. Conclusion: The clinical effect of Huayu Tongluo Decoction combined with edaravone injection in the treatment of patients with acute cerebral infarction is significant, which can further improve the clinical symptoms, improve neurological function and improve the quality of life.
\end{abstract}

\section{Introduction}

Cerebral infarction is a clinical multi-encephalic vascular disease, and its incidence accounts for about $75 \%$ of all cerebrovascular diseases, which can cause serious damage to the patient's nerve cells, resulting in high disability and mortality [1]. At present, the clinical use of statins, neurotrophic agents and antiplatelet drugs in the treatment of acute cerebral infarction, combined with free radical scavengers, can alleviate clinical symptoms to a certain extent, promote the recovery of neurological function, but the overall efficacy is not ideal [ 2]. In recent years, the combination of traditional Chinese and Western medicine has been gradually applied to the treatment of cerebral infarction, and the role of traditional Chinese medicine in promoting neurological improvement has gradually received clinical attention [3]. This study selected 86 patients with acute cerebral infarction in our hospital, and studied the clinical effects of Huayu Tongluo Decoction combined with edaravone injection. The report is as follows.

\section{Materials and Methods}

\subsection{General Information.}

86 patients with acute cerebral infarction received from our hospital from October 2016 to May 2018 were randomly divided into two groups, including 43 in the control group, 19 in female, and 24 in male; age $53 \sim 79$ years old, mean (64.52 \pm 5.43 ) years old; onset to admission time $2 \sim 9 \mathrm{~h}$, average $(5.13 \pm 1.26) \mathrm{h}$. The study group consisted of 43 patients, 21 females and 22 males; the age ranged from 52 to 77 years old, with an average of $(63.62 \pm 5.28)$ years old; the onset time to 
admission was 1 to 8 hours, with an average of $(4.97 \pm 1.15)$ hours. There was no significant difference in baseline data between the two groups $(\mathrm{P}>0.05)$. This study complies with the relevant requirements of the Helsinki Declaration of the World Medical Association.

\subsection{Selection Criteria.}

Inclusion of the relevant diagnostic criteria for acute phase of cerebral infarction [4]; no contraindications for this study; no cerebral hemorrhage; patients and their families are aware of this study, voluntarily joined.

It exclude those with a history of mental illness; patients with malignant tumors; severe liver and kidney dysfunction; severe heart and lung disease; poor treatment compliance.

\subsection{Methods.}

Both groups received routine treatment, including prevention of cerebral edema, administration of statins, neurotrophic drugs, aspirin, anti-infection, regulation of blood pressure and blood sugar, on the basis of different treatment options.

The control group was treated with edaravone injection (China Resources Shuanghe Pharmaceutical Co., Ltd., Guoyao Zhunzi H20130051), usage and dosage: edaravone injection 30 $\mathrm{mg}+$ normal saline $100 \mathrm{ml}$ intravenous infusion, 2 times $/ \mathrm{d}$.

The research group was treated with Huayu Tongluo Decoction combined with edaravone injection. Edaravone injection was the same as the control group, Huayu Tongluo Decoction: prescription: Danshen, Rhubarb, Pinellia, Tianma, Chuanniu Knee, sputum, tangerine peel, white atractylodes, phlegm, biliary star, scorpion yellow, silkworm, licorice; add water to cook, 1 dose / d, taken twice in the morning and evening. Both groups were treated for 14 days.

\subsection{Efficacy determination.}

The National Institutes of Health Neurological Deficit Score (NIHSS) was used to assess the degree of neurological deficits before and after treatment. The efficacy criteria were determined according to the NIHSS score and the degree of disability: basic recovery: NIHSS score Reduced $\geq 90 \%$, no dysfunction; markedly effective: $45 \% \leq$ NIHSS score decreased $<90 \%$, disability level 1-3; effective: $18 \% \leq$ NIHSS score decreased $<45 \%$; invalid: failed to meet the above criteria. Total effective rate $=($ basic recovery + marked effect + effective $) / 43 \times 100 \%$.

\subsection{Observation Indicators}

(1) treatment effect. (2) NIHSS score before and after treatment. (3) TCM syndrome scores before and after treatment, including main symptoms (biased numbness, hemiplegia, tongue slant), secondary symptoms (dizziness, strong tongue or nonsense), tongue veins (dark tongue, thin white tongue coating / White greasy, pulse string slip), according to the severity of the disease score, score 0 to 4 points, 0 points: none; 1 point: slight; 2 points: progress; 3 points: moderate; 4 points: severe; Scored 12 points, the total score of the secondary disease was 8 points, and the total score of the tongue and vein was 12 points. (4) Quality of life, assessed by the Spitzer Quality of Life Index (QLI), the higher the score, the better the quality of life.

1.6 Statistical methods Data processing using SPSS22.0 software, measurement data: expressed as $( \pm \mathrm{s}), \mathrm{t}$ test; count data: expressed as $\mathrm{n}(\%), \chi 2$ test; the difference was statistically significant $\mathrm{P}<$ 0.05 .

\section{Results}

Comparison of Curative Effect. The total effective rate of treatment group (95.35\%) was higher than that of the control group $(79.07 \%)(\mathrm{P}<0.05)$. See Table 1. 
Table 1 efficacy comparison n (\%)

\begin{tabular}{lllllll}
\hline Group & $\begin{array}{l}\text { Number } \\
\text { of cases }\end{array}$ & invalid & effective & $\begin{array}{l}\text { Significant } \\
\text { effect }\end{array}$ & $\begin{array}{l}\text { Basic } \\
\text { recovery }\end{array}$ & $\begin{array}{l}\text { Total } \\
\text { efficiency }\end{array}$ \\
\hline $\begin{array}{l}\text { research } \\
\text { group }\end{array}$ & 43 & $2(4.65)$ & $12(27.91)$ & $23(53.49)$ & $6(13.95)$ & $41(95.35)$ \\
Control & 43 & $9(20.93)$ & $14(32.56)$ & $18(41.86)$ & $2(4.65)$ & $34(79.07)$ \\
group & & & & & & 5.108 \\
$\chi^{2}$ & & & & & & 0.024 \\
$P$ & & & & & & \\
\hline
\end{tabular}

Before TCM syndrome treatment, there were no significant differences between the main symptoms, secondary diseases and tongue and pulse of the study group ( $\mathrm{P}>0.05)$. After treatment, the scores of main symptoms, secondary diseases and tongue and pulse of the two groups were compared. The treatment was reduced before treatment, and the study group was lower than the control group $(\mathrm{P}<0.05)$. See Table 2 .

Table 2 TCM syndrome points ( \pm s, points)

\begin{tabular}{|c|c|c|c|c|c|c|c|}
\hline \multirow[b]{2}{*}{ Group } & \multirow{2}{*}{$\begin{array}{l}\mathrm{Nu} \\
\text { mbe } \\
\mathrm{r}\end{array}$} & \multicolumn{2}{|c|}{ Main symptom } & \multicolumn{2}{|c|}{ Secondary disease } & \multicolumn{2}{|l|}{ Tongue } \\
\hline & & $\begin{array}{l}\text { Before } \\
\text { treatment }\end{array}$ & $\begin{array}{l}\text { After } \\
\text { treatment }\end{array}$ & $\begin{array}{l}\text { Before } \\
\text { treatment }\end{array}$ & $\begin{array}{l}\text { After } \\
\text { treatment }\end{array}$ & $\begin{array}{l}\text { Before } \\
\text { treatment }\end{array}$ & $\begin{array}{l}\text { After } \\
\text { treatment }\end{array}$ \\
\hline $\begin{array}{l}\text { research } \\
\text { group }\end{array}$ & 43 & $\begin{array}{l}10.43 \pm 1.3 \\
1\end{array}$ & $1.30 \pm 0.95^{\mathrm{a}}$ & $7.54 \pm 0.37$ & $1.42 \pm 0.85^{\mathrm{a}}$ & $\begin{array}{l}10.53 \pm 1.0 \\
8\end{array}$ & $1.31 \pm 0.95^{\mathrm{a}}$ \\
\hline $\begin{array}{l}\text { Control } \\
\text { group }\end{array}$ & 43 & $\begin{array}{l}10.29 \pm 1.2 \\
8\end{array}$ & $3.76 \pm 1.15^{\mathrm{a}}$ & $7.47 \pm 0.34$ & $3.52 \pm 1.06^{\mathrm{a}}$ & $\begin{array}{l}10.34 \pm 1.0 \\
3\end{array}$ & $3.86 \pm 1.16^{\mathrm{a}}$ \\
\hline$t$ & & 0.501 & 10.814 & 0.914 & 10.135 & 0.835 & 11.152 \\
\hline$P$ & & 0.618 & 0.000 & 0.634 & 0.000 & 0.406 & 0.000 \\
\hline
\end{tabular}

Note: Compared with the same group before treatment, $\mathrm{aP}<0.05$

Nerve function and quality of life Before treatment, the NIHSS and QLI scores of the study group were not significantly different from the control group $(\mathrm{P}>0.05)$. After treatment, the NIHSS and QLI scores of the two groups were improved compared with the pre-treatment, and the NIHSS score of the study group was higher than that of the control group. Low, QLI score was higher than the control group $(\mathrm{P}<0.05)$. See Table 3 .

Table 3 NIHSS and QLI scores ( \pm s, minutes)

\begin{tabular}{|c|c|c|c|c|c|c|c|c|c|}
\hline \multirow[b]{2}{*}{ Group } & \multirow[b]{2}{*}{ Number } & \multicolumn{2}{|l|}{ NIHSS } & \multirow[b]{2}{*}{$t$} & \multirow[b]{2}{*}{$P$} & \multicolumn{2}{|l|}{ QLI } & \multirow[b]{2}{*}{$t$} & \multirow[b]{2}{*}{$P$} \\
\hline & & $\begin{array}{l}\text { Before } \\
\text { treatment }\end{array}$ & $\begin{array}{l}\text { After } \\
\text { treatment }\end{array}$ & & & $\begin{array}{l}\text { Before } \\
\text { treatment }\end{array}$ & $\begin{array}{l}\text { After } \\
\text { treatment }\end{array}$ & & \\
\hline $\begin{array}{l}\text { research } \\
\text { group }\end{array}$ & 43 & $22.33 \pm 2.12$ & $9.37 \pm 2.06$ & 28.750 & 0.000 & $5.08 \pm 0.97$ & $7.21 \pm 1.05$ & 9.771 & 0.000 \\
\hline $\begin{array}{l}\text { Control } \\
\text { group }\end{array}$ & 43 & $21.95 \pm 2.08$ & $11.67 \pm 2.18$ & 22.372 & 0.000 & $5.16 \pm 1.10$ & $6.24 \pm 0.95$ & 4.873 & 0.000 \\
\hline$t$ & & 0.817 & 5.029 & & & 0.358 & 4.492 & & \\
\hline$P$ & & 0.416 & 0.000 & & & 0.722 & 0.000 & & \\
\hline
\end{tabular}

\section{Discussion}

After the onset of cerebral infarction, the brain tissue is in an ischemic and hypoxic state, and the energy metabolism is abnormal, resulting in the mass production of oxygen free radicals, which can aggravate the damage of brain tissue and nerve cells. Edaravone injection is an oxygen free radical scavenger, which can be used for lipids. The peroxidation produces an inhibitory effect, scavenging oxygen free radicals, thereby reducing brain tissue damage [5]. The total effective rate of treatment 
with edaravone injection in this study was $79.07 \%$, which showed that the curative effect was good, but there is still room for improvement.

Traditional Chinese medicine belongs to the category of "stroke" in cerebral infarction. It believes that its pathogenesis is qi and blood, and the brain and sputum are mutually obstructed, causing the disease to rise and fall due to dysfunction, which requires the treatment of blood circulation, dampness and dampness [6]. Huayu Tongluo Decoction consists of traditional Chinese medicines such as Salvia miltiorrhiza, Rhubarb, Pinellia, Tianma, Sichuan Achyranthes, Poria, Chenpi, Shengbai, Zhishi, Dannanxing, Tianzhuhuang, silkworm, and licorice [7]. In this study, Huayu Tongluo Decoction combined with edaravone injection was used in the treatment of patients with acute cerebral infarction. The results showed that the total effective rate of the study group was as high as $95.35 \%$, which was significantly higher than that of the control group, and the NIHSS score of the study group. It can be seen that the addition of Huayu Tongluo Decoction can further improve the neurological function of patients and enhance the curative effect. Modern pharmacological studies have shown that in the Huayu Tongluo Decoction, Salvia miltiorrhiza has the functions of dilating the coronary artery, improving the ability of cells to resist hypoxia, and inhibiting platelet aggregation; rhubarb has the function of promoting blood circulation; The role of platelet aggregation, at the same time can resist the excitability of amino acid toxicity, relieve brain tissue edema caused by ischemia; Gastrodia has the effect of dilating blood vessels, can reduce cerebral vascular resistance, and can play an analgesic effect; Pinellia, tangerine peel, etc. have certain The role of regulating blood pressure helps to improve the condition [8]. Therefore, Huatan Tongluo Decoction can improve brain microcirculation, inhibit platelet aggregation, improve blood rheology, thereby promoting recovery of nerve function and enhancing efficacy. According to the results of this study, after treatment, the scores of main symptoms, secondary diseases and tongue and pulse of the study group were lower than those of the control group, and the QLI score was higher than that of the control group $(\mathrm{P}<0.05)$. It shows that the above drug regimen can further improve clinical symptoms and improve the quality of life of patients.

In summary, the treatment of acute cerebral infarction patients with Huayu Tongluo Decoction combined with edaravone injection can significantly improve the treatment effect, can further improve the clinical symptoms of patients, improve neurological function, improve quality of life, and is worthy of clinical promotion.

\section{References}

[1] Qiu Chenfeng, Feng Xuewen, Wu Weilin, et al. The efficacy and mechanism of Yuriklin and Edaravone in patients with acute cerebral infarction [J]. Chinese Journal of General Practice, 2016, 14(8): 1319- 1321.

[2] Wang Dongyu. Clinical study of Yishen Dingxuan Decoction combined with edaravone in the treatment of acute cerebral infarction[J].Henan Traditional Chinese Medicine, 2015, 35(12): 2949-2950.

[3] Zhang Chenhao, Gao Kun, Qiao Baohong, et al. Efficacy of Yidalafeng combined with Qufeng Huayu Tongluo Decoction in the treatment of acute cerebral infarction with wind-stagnation and collaterals[J].Modern Journal of Integrated Traditional Chinese and Western Medicine, 2016,25(7):753-754.

[4] Chinese Medical Association Physical Medicine and Rehabilitation Society, China's cerebral infarction acute rehabilitation expert consensus group. Chinese cerebral infarction acute rehabilitation expert consensus [J]. Chinese Journal of Physical Medicine and Rehabilitation, 2016, 38 (1): $1-6$.

[5] Cao Xinchao. Clinical effect of edaravone combined with Qifeng Huayu Tongluo Decoction in the treatment of acute cerebral infarction with wind-stagnation and collaterals [J].Journal of Clinical Medicine Research and Practice, 2018,2(6):119-120. 
[6] Zheng Zhucheng. Effect of Tongqiao Huoxue Decoction combined with edaravone injection in the treatment of acute cerebral infarction [J]. Chinese Folk Therapy, 2017, 25(11): 59-60.

[7] Zhou Yuting.Effects and prognosis of Yidalafeng combined with Qifeng Huayu Tongluo Decoction on patients with acute cerebral infarction with wind-stagnation and collaterals [J].Chinese Journal of Convalescent Medicine, 2017,26(6):636-638.

[8] Hu Shuyun, Chen Li, Jin Hua. Efficacy of Yidalafeng combined with Qufeng Huayu Tongluo Decoction on patients with acute cerebral infarction with wind-stagnation and collaterals [J]. Chinese contemporary medicine, 2017,24(2):144 -146. 